\title{
Supersymmetric bulk-brane coupling with odd gauge fields
}

\author{
Dmitry V. Belyaev \\ Deutsches Elektronen-Synchrotron \\ DESY-Theory, Notkestrasse 85 \\ 22603 Hamburg, Germany \\ E-mail: dmitry.belyaev@desy.de
}

\begin{abstract}
Supersymmetric bulk-brane coupling in Horava-Witten and Randall-Sundrum scenarios, when considered in the orbifold ("upstairs") picture, enjoys similar features: a modified Bianchi identity and a modified supersymmetry transformation for the "orthogonal" part of the gauge field. Using a toy model with a 5D vector multiplet in the bulk (like in Mirabelli-Peskin model, but with an odd gauge field $A_{m}$ ), we explain how these features arise from the superfield formulation. We also show that the corresponding construction in the boundary ("downstairs") picture requires introduction of a special "compensator" (super)field.
\end{abstract}




\section{Contents}

1. Introduction 2

2. Mirabelli-Peskin model with odd $A_{m}$

$2.15 \mathrm{D}$ vector multiplet

$2.2 \mathrm{OP}, \mathrm{BP}$ and $N=1$ supersymmetry

$2.3 \quad N=1$ superfields $\mathbf{V}_{\mathbf{2}}$ and $\boldsymbol{\Phi}_{\mathbf{2}}$

2.4 Superfield Lagrangian 5

3. Bulk-brane coupling in superfields 6

3.1 Boundary picture 6

3.2 OP with singular $\boldsymbol{\Phi}_{\mathbf{2}}$

3.3 OP with non-singular $\boldsymbol{\Phi}_{\mathbf{2}}$

4. Bulk-brane coupling in components 10

4.1 Boundary conditions 10

4.2 Compensator (super)field 11

4.3 Boundary picture 11

4.4 OP with singular fields 12

4.5 OP with singular $A_{5}$

4.6 OP with non-singular fields 15

5. On-shell coupling 15

5.1 Modified Bianchi identity 16

5.2 Boundary picture 16

5.3 Orbifold picture 17

5.4 Example 18

6. Summary and Conclusions 19

7. Appendix 20

A Superfield components 20

B $\quad \mathbf{V}_{\mathbf{2}}$ and $\boldsymbol{\Phi}_{\mathbf{2}}$ without WZ

9 Boundary conditions for supersymmetry 23 


\section{Introduction}

The last decade saw a revival of interest in theories with extra dimensions and brane-world scenarios. In 1996, Horava and Witten [1] showed that 11D supergravity on a manifold with boundary (or on $S^{1} / \mathbb{Z}_{2}$ orbifold) arises as a low energy limit of the strongly coupled heterotic string theory. Three years later, Randall and Sundrum [2] demonstrated that a simpler 5D construction with a cosmological constant in the bulk is sufficient to naturally solve the gauge hierarchy problem and leads to interesting phenomenological consequences.

A minimal supersymmetric version of the Randall-Sundrum scenario, with just the ten-

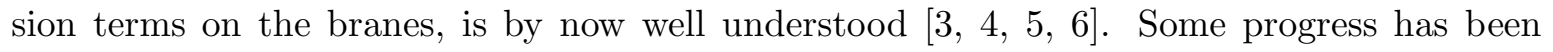
made in including additional matter fields on the branes (see ref. [7] and references therein), but the construction is not yet complete. One interesting observation of ref. [0] is that the supersymmetric bulk-brane coupling (in the orbifold picture) in both the 11D and 5D cases has two basic features in common:

1) the field strength of the bulk gauge field is shifted so that it satisfies a modified Bianchi identity; and

2) supersymmetry transformation of the "orthogonal" component of the gauge field $\left(C_{11 A B}\right.$ in $11 \mathrm{D}$ and $B_{5}$ in $5 \mathrm{D}$ ) is modified accordingly.

Besides these modifications, the simplest version of the coupling (to the 2-Fermi order) requires only adding the Noether coupling term to the free brane Lagrangian.

Supersymmetric bulk-brane coupling can be nicely formulated using $4 \mathrm{D}$ superfields. The original idea is due to Mirabelli and Peskin [8] (who worked with supermultiplets instead of superfields); in the superfield language, the method was developed and generalized to dimensions higher than five in ref. [9]. Although this method has already been widely used, the basic features of the bulk-brane coupling listed above have not yet been explained by it. In this paper, we will fill in the gap.

In our discussion, we will use the toy (globally supersymmetric) model of Mirabelli and Peskin, with an abelian 5D vector multiplet in the bulk. In the orbifold picture, the 4D vector $\left(A_{m}\right)$ and the $4 \mathrm{D}$ scalar $\left(A_{5}\right)$ components of the gauge field $A_{M}$ have opposite parities. Instead of choosing $A_{m}$ to be even, as in ref. [8], we will choose it to be odd to make contact with the supergravity constructions (where $C_{A B C}$ in $11 \mathrm{D}$ and $B_{m}$ in $5 \mathrm{D}$ are odd). This also flips the parities of the $4 \mathrm{D}$ superfields used to describe the $5 \mathrm{D}$ vector multiplet, compared to refs. 8, 9]. We will find that this model reproduces the features of the supergravity bulk-brane coupling surprisingly well.

Our key results are as follows. In the orbifold picture (OP), recovering the right component structure of the coupling from its superfield form requires a certain field redefinition that makes all bulk fields except $A_{5}$ non-singular. In the boundary picture (BP), the singularity of $A_{5}$ is replaced by the presence of a special boundary compensator $K$. In both cases, the boundary condition on the odd part of the gauge field is $A_{m}=J_{m}$, where $J_{m}$ is a function 
of the brane/boundary fields. This boundary condition is required for supersymmetry of the action in the BP, but not in the OP.

The structure of the paper is best seen from the Contents. We note here only that the agreement with the supergravity constructions is achieved in Section 4.5, and more explicitly in the example of Section 5.4 .

\section{Mirabelli-Peskin model with odd $A_{m}$}

In this section, we review the essentials of the $5 \mathrm{D}$ gauge supermultiplet, both in the component formulation and using 4D $N=1$ superfields; $\mathrm{OP}$ and $\mathrm{BP}$ are defined here. Our conventions are the same as in ref. [11]; supersymmetry conventions follow closely those of Wess and Bagger [13].

\subsection{D vector multiplet}

The abelian 5D gauge supermultiplet consists of a gauge field $A_{M}(M=0,1,2,3,5)$, a real scalar $\Phi$, a symplectic-Majorana spinor (gaugino) $\Lambda_{i}(i=1,2)$, and a triplet of real auxiliary fields $X_{a}(a=1,2,3)$. The Lagrangian for this multiplet is

$$
\mathcal{L}_{5}=-\frac{1}{4} F_{M N} F^{M N}-\frac{1}{2} \partial_{M} \Phi \partial^{M} \Phi-\frac{i}{2} \widetilde{\Lambda}^{i} \Gamma^{M} \partial_{M} \Lambda_{i}+\frac{1}{2} X_{a} X_{a}
$$

The corresponding supersymmetry transformations are

$$
\begin{aligned}
\delta_{\mathcal{H}} A_{M} & =i \widetilde{\mathcal{H}}^{i} \Gamma_{M} \Lambda_{i} \\
\delta_{\mathcal{H}} \Phi & =i \widetilde{\mathcal{H}}^{i} \Lambda_{i} \\
\delta_{\mathcal{H}} X_{a} & =\widetilde{\mathcal{H}}^{i}\left(\sigma_{a}\right)_{i}{ }^{j} \Gamma^{M} \partial_{M} \Lambda_{j} \\
\delta_{\mathcal{H}} \Lambda_{i} & =\delta_{\mathcal{H}}^{\prime} \Lambda_{i}+\delta_{\mathcal{H}}^{\prime \prime} \Lambda_{i},
\end{aligned}
$$

where we made the following split,

$$
\delta_{\mathcal{H}}^{\prime} \Lambda_{i}=\left(\Sigma^{M N} F_{M N}+\Gamma^{M} \partial_{M} \Phi\right) \mathcal{H}_{i}, \quad \delta_{\mathcal{H}}^{\prime \prime} \lambda_{i}=X_{a}\left(\sigma_{a}\right)_{i}{ }^{j} \mathcal{H}_{j},
$$

separating out the auxiliary part of the transformation. (The supersymmetry parameter $\mathcal{H}_{i}$ is a constant symplectic-Majorana spinor.) Under the supersymmetry transformations, the Lagrangian varies into a total derivative. This is to be compared with the general variation, when one also finds a total derivative plus terms that vanish only when equations of motion $(\mathrm{EOM})$ are used. The total derivatives in these two cases are similar, but differ in the fermionic parts. In the case at hand, we find

$$
\delta \mathcal{L}_{5}=(\mathrm{EOM})+\partial_{M} K^{M}, \quad \delta_{\mathcal{H}} \mathcal{L}_{5}=\partial_{M} \widetilde{K}^{M},
$$

where

$$
\begin{aligned}
& K^{M}=-F^{M N} \delta A_{N}-\delta \Phi \partial^{M} \Phi-\frac{i}{2} \widetilde{\Lambda}^{i} \Gamma^{M} \delta \Lambda^{i} \\
& \widetilde{K}^{M}=-F^{M N} \delta_{\mathcal{H}} A_{N}-\delta_{\mathcal{H}} \Phi \partial^{M} \Phi+\frac{i}{2} \widetilde{\Lambda}^{i} \Gamma^{M} \delta_{\mathcal{H}}^{\prime} \Lambda_{i}-\frac{i}{2} \widetilde{\Lambda}^{i} \Gamma^{M} \delta_{\mathcal{H}}^{\prime \prime} \Lambda_{i} .
\end{aligned}
$$

The total derivatives are irrelevant on the orbifold, but essential in the boundary picture. 


\subsection{OP, BP and $N=1$ supersymmetry}

In the orbifold picture (OP), the $5 \mathrm{D}$ space is $\mathbb{R}^{1,4}$ with a $\mathbb{Z}_{2}$ symmetry realized as a reflection $x^{5} \equiv z \rightarrow-z$. The "fixed point" at $z=0$ is a $4 \mathrm{D}$ plane that we call a "brane". In the boundary picture $(\mathrm{BP})$, the $5 \mathrm{D}$ space is $\mathcal{M}=\mathbb{R}^{1,3} \times[0,+\infty)$, with boundary at $z=0$. In both cases, it is convenient to make the " $5 \rightarrow 4$ " split, using $M=\{m, 5\}(m=0,1,2,3)$, and to convert symplectic-Majorana spinors into pairs of two-component spinors: $\Lambda_{i} \rightarrow\left(\lambda_{1}, \lambda_{2}\right)$, $\mathcal{H}_{i} \rightarrow\left(\eta_{1}, \eta_{2}\right)$. This leads to the following form of the Lagrangian, ${ }^{1}$

$$
\begin{aligned}
\mathcal{L}_{5}= & -\frac{1}{4} F_{m n} F^{m n}-\frac{1}{2} F_{m 5} F^{m 5}-\frac{1}{2} \partial_{m} \Phi \partial^{m} \Phi-\frac{1}{2} \partial_{5} \Phi \partial_{5} \Phi+\frac{1}{2} X_{12} X_{12}^{*}+\frac{1}{2} X_{3}^{2} \\
& -\left[\frac{i}{2} \lambda_{1} \sigma^{m} \partial_{m} \bar{\lambda}_{1}+\frac{i}{2} \lambda_{2} \sigma^{m} \partial_{m} \bar{\lambda}_{2}+\frac{1}{2}\left(\lambda_{2} \partial_{5} \lambda_{1}-\lambda_{1} \partial_{5} \lambda_{2}\right)+\text { h.c. }\right] .
\end{aligned}
$$

When a brane/boundary is present, we can preserve only a half of the $N=2$ supersymmetry parametrized by $\eta_{1}$ and $\eta_{2}$. Without loss of generality, we set

$$
\eta_{1}=\eta, \quad \eta_{2}=0
$$

This gives the following $N=1$ supersymmetry transformations,

$$
\begin{aligned}
\delta_{\eta} A_{m} & =i \eta \sigma_{m} \bar{\lambda}_{1}+h . c . \\
\delta_{\eta} A_{5} & =-\eta \lambda_{2}+h . c . \\
\delta_{\eta} \Phi & =-i \eta \lambda_{2}+h . c . \\
\delta_{\eta} \lambda_{1} & =\sigma^{m n} \eta F_{m n}+i\left(X_{3}-\partial_{5} \Phi\right) \eta \\
\delta_{\eta} \lambda_{2} & =-i \sigma^{m} \bar{\eta} F_{m 5}-\sigma^{m} \bar{\eta} \partial_{m} \Phi-i X_{12} \eta \\
\delta_{\eta} X_{12} & =2 i \bar{\eta} \partial_{5} \bar{\lambda}_{1}-2 \overline{\eta \sigma}^{m} \partial_{m} \lambda_{2} \\
\delta_{\eta} X_{3} & =-i \eta \partial_{5} \lambda_{2}-\eta \sigma^{m} \partial_{m} \bar{\lambda}_{1}+\text { h.c. }
\end{aligned}
$$

In the orbifold picture, we must choose parity assignments for all fields and parameters. We are interested in the case when $\eta_{1}$ is even and $A_{m}$ is odd, which leads to the following set of assignments, ${ }^{2}$

$$
\text { even : } A_{5}, \Phi, \lambda_{2}, X_{12}, \eta_{1} \quad \text { odd : } A_{m}, \lambda_{1}, X_{3}, \eta_{2} \text {. }
$$

With these assignments, the Lagrangian is even, whereas the equations of motion and supersymmetry transformations are parity covariant.

\footnotetext{
${ }^{1}$ In our notation, $F_{m n}=\partial_{m} A_{n}-\partial_{n} A_{m}, F_{m 5}=\partial_{m} A_{5}-\partial_{5} A_{m}, X_{12}=X_{1}+i X_{2}$.

${ }^{2}$ Under the $\mathbb{Z}_{2}$ reflection, each field $f(x, z)$ is mapped into $f(x,-z)=P[f] f(x,+z)$ with $P[f]= \pm 1$. We call $P[f]=+1$ fields "even" and $P[f]=-1$ fields "odd".
} 


\section{$2.3 N=1$ superfields $\mathbf{V}_{2}$ and $\Phi_{2}$}

$N=1$ supersymmetry is most conveniently described in terms of $N=1$ superfields. For the $5 \mathrm{D}$ vector multiplet, we need two $4 \mathrm{D} N=1$ superfields: a gauge superfield $\mathbf{V}_{\mathbf{2}}$ and a chiral superfield $\mathbf{\Phi}_{\mathbf{2}} .{ }^{3}$ If we take $\mathbf{V}_{\mathbf{2}}$ in the Wess-Zumino (WZ) gauge (see Appendix B for a discussion of this choice), then the relation between the component bulk fields and components of the superfields is given by [9, 10]

$$
\begin{aligned}
& \mathbf{V}_{\mathbf{2}}=\left(0, \quad 0, \quad 0 ; \quad A_{m}, \quad \lambda_{1}, \quad X_{3}-\partial_{5} \Phi\right) \\
& \boldsymbol{\Phi}_{\mathbf{2}}=\left(\Phi+i A_{5}, \quad-i \sqrt{2} \lambda_{2}, \quad-X_{12}\right) .
\end{aligned}
$$

Here and henceforth we represent superfields by listing their components in a definite order (see Appendix A). The supersymmetry transformations (2.8) are reproduced by the following superfield transformations,

$$
\begin{aligned}
\delta_{\eta} \mathbf{V}_{\mathbf{2}} & =(\eta Q+\bar{\eta} \bar{Q}) \mathbf{V}_{\mathbf{2}}+\boldsymbol{\Lambda}_{\mathbf{2}}(\eta)+\boldsymbol{\Lambda}_{\mathbf{2}}(\eta)^{\dagger} \\
\delta_{\eta} \mathbf{\Phi}_{\mathbf{2}} & =(\eta Q+\bar{\eta} \bar{Q}) \mathbf{\Phi}_{\mathbf{2}}+2 \partial_{5} \boldsymbol{\Lambda}_{\mathbf{2}}(\eta)
\end{aligned}
$$

where the compensating gauge transformation (keeping $\mathbf{V}_{\mathbf{2}}$ in the $\mathrm{WZ}$ gauge) is given by

$$
\boldsymbol{\Lambda}_{\mathbf{2}}(\eta)=\left(0, \quad \frac{1}{\sqrt{2}} \sigma^{m} \bar{\eta} A_{m}, \quad-i \bar{\eta} \bar{\lambda}_{1}\right)
$$

Similarly, the bulk $U(1)$ gauge transformation,

$$
\delta_{u} A_{M}=\partial_{M} u \quad \Leftrightarrow \quad \delta_{u} A_{m}=\partial_{m} u, \quad \delta_{u} A_{5}=\partial_{5} u,
$$

is reproduced by the superfield gauge transformation

$$
\delta_{u} \mathbf{V}_{\mathbf{2}}=\boldsymbol{\Lambda}_{\mathbf{2}}(u)+\boldsymbol{\Lambda}_{\mathbf{2}}(u)^{\dagger}, \quad \delta_{u} \boldsymbol{\Phi}_{\mathbf{2}}=2 \partial_{5} \boldsymbol{\Lambda}_{\mathbf{2}}(u)
$$

with the following parameter,

$$
\mathbf{\Lambda}_{\mathbf{2}}(u)=\left(\frac{i}{2} u, 0,0\right)
$$

\subsection{Superfield Lagrangian}

The Lagrangian $\mathcal{L}_{5}$ is gauge invariant and, therefore, should be constructed out of gauge invariant superfields. Two basic gauge invariant superfields are ${ }^{4}$

$$
\mathbf{Z}_{\mathbf{2}}=\partial_{5} \mathbf{V}_{\mathbf{2}}-\frac{1}{2}\left(\boldsymbol{\Phi}_{\mathbf{2}}+\mathbf{\Phi}_{\mathbf{2}}^{\dagger}\right), \quad \eta \mathbf{W}_{\mathbf{2}}=-\frac{1}{4} \eta^{\alpha} \overline{D D} D_{\alpha} \mathbf{V}_{\mathbf{2}}
$$

\footnotetext{
${ }^{3}$ The subscript "2" on $\mathbf{V}_{\mathbf{2}}$ and $\boldsymbol{\Phi}_{\mathbf{2}}$ indicates that these are bulk superfields. We reserve $\mathbf{V}$ and $\boldsymbol{\Phi}$ for denoting brane-localized superfields.

${ }^{4}$ We hide the spinor index $\alpha$ on $\mathbf{W}_{\mathbf{2}}$ by contracting it with another spinor. For the definition of the supersymmetry operator $Q_{\alpha}$ and the covariant superspace derivative $D_{\alpha}$, see ref. [13].
} 
Their components are related to the bulk fields in the following way,

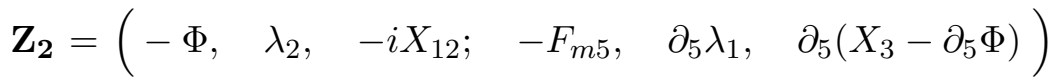

$$
\begin{aligned}
& \eta \mathbf{W}_{\mathbf{2}}=\left(-i \eta \lambda_{1}, \quad \frac{1}{\sqrt{2}}\left[\eta\left(X_{3}-\partial_{5} \Phi\right)+i \sigma^{m n} \eta F_{m n}\right], \quad \eta \sigma^{m} \partial_{m} \bar{\lambda}_{1}\right) .
\end{aligned}
$$

The superfield Lagrangian that reproduces $\mathcal{L}_{5}$ up to a total derivative is given by

$$
\begin{aligned}
\mathcal{L}_{5}^{\prime} & =\frac{1}{4} \int d^{2} \theta \mathbf{W}_{\mathbf{2}}{ }^{2}+\text { h.c. }+\int d^{2} \theta d^{2} \bar{\theta} \mathbf{Z}_{\mathbf{2}}{ }^{2} \\
& =\int d^{2} \theta d^{2} \bar{\theta}\left[\frac{1}{8} \mathbf{V}_{\mathbf{2}} D^{\alpha} \overline{D D} D_{\alpha} \mathbf{V}_{\mathbf{2}}+\mathbf{Z}_{\mathbf{2}}{ }^{2}\right],
\end{aligned}
$$

where the second form is particularly suited for deriving superfield equations of motion (and boundary conditions) and allows us to omit the overall superspace integration. ${ }^{5}$ Writing $\mathcal{L}_{5}^{\prime}$ in components and comparing with $\mathcal{L}_{5}$, eq. (2.6), we find

$$
\mathcal{L}_{5}^{\prime}=\mathcal{L}_{5}-\partial_{5} Y^{\prime}, \quad Y^{\prime}=\Phi\left(X_{3}-\partial_{5} \Phi\right)+\frac{1}{2}\left(\lambda_{1} \lambda_{2}+\text { h.c. }\right) .
$$

In terms of the actions, on a manifold with boundary $\mathcal{M}=\mathbb{R}^{1,3} \times[0,+\infty)$, we have

$$
S_{5}^{\prime}=\int_{\mathcal{M}} \mathcal{L}_{5}^{\prime}, \quad S_{5}=\int_{\mathcal{M}} \mathcal{L}_{5} \Rightarrow S_{5}^{\prime}=S_{5}+\int_{\partial \mathcal{M}} Y^{\prime}
$$

\section{Bulk-brane coupling in superfields}

In this section, we construct supersymmetric coupling of the bulk $5 \mathrm{D}$ gauge multiplet to the brane/boundary. The coupling gives rise to a boundary condition $\mathbf{V}_{\mathbf{2}} \stackrel{+0}{=} \mathbf{J}$, where $\mathbf{J}$ is a function of brane localized superfields $\mathbf{V}, \mathbf{\Phi}$, and a special compensator superfield $\mathbf{K}$ that, in the orbifold picture, corresponds to the singular part of $\mathbf{\Phi}_{\mathbf{2}}$.

\subsection{Boundary picture}

In the previous section, we arrived at the following superfield action on a manifold with boundary $\mathcal{M}$,

$$
S_{5}^{\prime}=\int_{\mathcal{M}}\left[\frac{1}{8} \mathbf{V}_{\mathbf{2}} D \bar{D}^{2} D \mathbf{V}_{\mathbf{2}}+\mathbf{Z}_{\mathbf{2}}^{2}\right]
$$

Its general variation gives

$$
\delta S_{5}^{\prime}=\int_{\mathcal{M}}\left[-\mathbf{Z}_{\mathbf{2}} \delta \mathbf{\Phi}_{\mathbf{2}}+\text { h.c. }+\left(\frac{1}{4} D \bar{D}^{2} D \mathbf{V}_{\mathbf{2}}-2 \partial_{5} \mathbf{Z}_{\mathbf{2}}\right) \delta \mathbf{V}_{\mathbf{2}}\right]-\int_{\partial \mathcal{M}} 2 \mathbf{Z}_{\mathbf{2}} \delta \mathbf{V}_{\mathbf{2}}
$$

\footnotetext{
${ }^{5}$ The superspace integral $\int d^{2} \theta d^{2} \bar{\theta}$ is implicit in the expressions for actions and Lagrangians in the rest of the paper. Note also that we omit total $\partial_{m}$ derivatives, as they are irrelevant in both the orbifold and boundary pictures. Total $\partial_{5}$ derivatives, however, are kept.
} 
The bulk equations of motion, therefore, are ${ }^{6}$

$$
\overline{D D} \mathbf{Z}_{2}=0, \quad \frac{1}{4} D \bar{D}^{2} D \mathbf{V}_{\mathbf{2}}-2 \partial_{5} \mathbf{Z}_{2}=0
$$

while the natural boundary condition, obtained by requiring the boundary piece of $\delta S_{5}^{\prime}$ to vanish for arbitrary $\delta \mathbf{V}_{\mathbf{2}}$, is 7

$$
\mathbf{Z}_{\mathbf{2}} \stackrel{+0}{=} 0
$$

This is the reason why $S_{5}^{\prime}$ is the right action for lifting on the orbifold with odd $\mathbf{Z}_{\mathbf{2}}$ (that is, with even $\mathbf{V}_{\mathbf{2}}$ and odd $\boldsymbol{\Phi}_{\mathbf{2}}$ ). Coupling bulk fields to brane localized matter would make this boundary condition inhomogeneous [11].

In this paper, we are interested in the other case, when $\mathbf{V}_{\mathbf{2}}$ is odd and $\mathbf{\Phi}_{\mathbf{2}}$ is even (therefore, $\mathbf{Z}_{\mathbf{2}}$ is even). The appropriate action is easy to guess. We define

$$
S_{5}^{\prime \prime}=S_{5}^{\prime}+\int_{\partial \mathcal{M}} 2 \mathbf{Z}_{2} \mathbf{V}_{2}
$$

Its general variation gives the same equations of motion in the bulk, but the boundary term and, therefore, the natural boundary condition are now different:

$$
\delta S_{5}^{\prime \prime}=(\mathrm{EOM})+\int_{\partial \mathcal{M}} 2 \mathbf{V}_{\mathbf{2}} \delta \mathbf{Z}_{\mathbf{2}} \Rightarrow \mathbf{V}_{\mathbf{2}} \stackrel{+0}{=} 0
$$

This shows that $S_{5}^{\prime \prime}$ is the right action for lifting on the orbifold with odd $\mathbf{V}_{\mathbf{2}}$.

Adding boundary interaction that leads to the boundary condition $\mathbf{V}_{\mathbf{2}} \stackrel{+0}{=} \mathbf{J}$ is now straightforward. For the complete bulk-plus-boundary action we take

$$
\begin{aligned}
S & =S_{5}^{\prime \prime}+\frac{1}{2} \int_{\partial \mathcal{M}} \mathcal{L}_{4}-\int_{\partial \mathcal{M}} 2 \mathbf{Z}_{\mathbf{2}} \mathbf{J} \\
& =S_{5}^{\prime}+\frac{1}{2} \int_{\partial \mathcal{M}} \mathcal{L}_{4}+\int_{\partial \mathcal{M}} 2 \mathbf{Z}_{\mathbf{2}}\left(\mathbf{V}_{\mathbf{2}}-\mathbf{J}\right),
\end{aligned}
$$

where $\mathcal{L}_{4}$ is a part of the boundary Lagrangian that does not depend on the bulk fields and is supersymmetric on its own. The general variation of the action gives the required boundary condition:

$$
\delta S=(\mathrm{EOM})+\int_{\partial \mathcal{M}} 2\left(\mathbf{V}_{\mathbf{2}}-\mathbf{J}\right) \delta \mathbf{Z}_{\mathbf{2}} \Rightarrow \mathbf{V}_{\mathbf{2}} \stackrel{+0}{=} \mathbf{J}
$$

However, despite being written in terms of superfields, the action is not yet guaranteed to be supersymmetric. Supersymmetry transformations of $\mathbf{V}_{\mathbf{2}}$ and $\mathbf{\Phi}_{\mathbf{2}}$, given in eq. (2.11), are a

\footnotetext{
${ }^{6}$ Equations of motion for chiral superfields are found by applying $\overline{D D}$ to what comes out from the general variation. See ref. [13] for more details.

${ }^{7}$ The symbol $\stackrel{+0}{=}$ is used to denote boundary conditions in both the boundary and orbifold pictures. In the orbifold picture, it means "on the positive side of the brane", at $z=+0$.
} 
combination of the standard piece (with the linear supersymmetry operator acting on them) and a special gauge transformation. As a result, the action can be supersymmetric only when it is gauge invariant. With $\mathbf{V}_{\mathbf{2}}$ appearing in the action explicitly, this can be achieved only if the gauge and supersymmetry transformations of $\mathbf{J}$ match those of $\mathbf{V}_{\mathbf{2}}$. That is, the action is supersymmetric provided $\mathbf{J}$ transforms as follows,

$$
\delta_{u} \mathbf{J}=\boldsymbol{\Lambda}_{\mathbf{2}}^{(+)}(u)+\boldsymbol{\Lambda}_{\mathbf{2}}^{(+)}(u)^{\dagger}, \quad \delta_{\eta} \mathbf{J}=(\eta Q+\bar{\eta} \bar{Q}) \mathbf{J}+\boldsymbol{\Lambda}_{\mathbf{2}}^{(+)}(\eta)+\boldsymbol{\Lambda}_{\mathbf{2}}^{(+)}(\eta)^{\dagger},
$$

where the superscript "(+)" indicates restriction of the bulk quantity to the boundary.

One can construct $\mathbf{J}=\mathbf{J}(\mathbf{V}, \boldsymbol{\Phi})$ with the above transformation laws. However, this inevitably requires relating bulk and boundary gauge invariances and leads to a rather strange form of the coupling. Another way to satisfy eq. (3.9), motivated by the orbifold picture construction (see below), is to introduce a special boundary superfield $\mathbf{K}$ with the following transformation properties,

$$
\delta_{u} \mathbf{K}=\mathbf{\Lambda}_{\mathbf{2}}^{(+)}(u), \quad \delta_{\eta} \mathbf{K}=(\eta Q+\bar{\eta} \bar{Q}) \mathbf{K}+\mathbf{\Lambda}_{\mathbf{2}}^{(+)}(\eta) .
$$

If we now define

$$
\mathbf{J}=\mathbf{K}+\mathbf{K}^{\dagger}+\mathbf{G}
$$

with $\mathbf{G}=\mathbf{G}(\mathbf{V}, \mathbf{\Phi})$ transforming as a gauge invariant quantity,

$$
\delta_{u} \mathbf{G}=0, \quad \delta_{\eta} \mathbf{G}=(\eta Q+\bar{\eta} \bar{Q}) \mathbf{G},
$$

then $\mathbf{J}$ transforms precisely as in eq. (3.9). This way we do not need to relate the bulk gauge transformation to a boundary one, which means that introducing the superfield $\mathbf{K}$ increases gauge symmetry of the action. Therefore, we can call $\mathbf{K}$ a "compensator" superfield.

With the superfield $\mathbf{K}$ present, we do not need a boundary gauge transformation, so that, for example, $\mathbf{G}=\boldsymbol{\Phi}^{\dagger} \boldsymbol{\Phi}$ is a valid choice. Note also that $\mathbf{K}$ does not appear in $\mathcal{L}_{4}$, but comes only with $\mathbf{J}$. As a result, its equation of motion is

$$
\overline{D D} \mathbf{Z}_{\mathbf{2}} \stackrel{+0}{=} 0 .
$$

As this coincides with the restriction of the bulk equation of motion for $\boldsymbol{\Phi}_{\mathbf{2}}$, eq. (3.3), to the boundary, our construction is consistent.

\subsection{OP with singular $\Phi_{2}$}

In the orbifold picture, the bulk-plus-brane Lagrangian, corresponding to the bulk-plusboundary action (3.7), turns out to be given by

$$
\mathcal{L}=\frac{1}{8} \mathbf{V}_{\mathbf{2}} D \bar{D}^{2} D \mathbf{V}_{\mathbf{2}}+\left[\mathbf{Z}_{\mathbf{2}}-2 \mathbf{G} \delta(z)\right]^{2}+\mathcal{L}_{4} \delta(z)
$$


The first part of it, explicitly showing $\mathbf{V}_{\mathbf{2}}$, is gauge invariant (up to a total $\partial_{m}$ derivative). As $\mathbf{Z}_{\mathbf{2}}$ is gauge invariant, the brane-localized term $\mathbf{G}$ must also be invariant under the bulk gauge transformation for the Lagrangian to be supersymmetric.

The full square structure of the interaction is required to guarantee that equations of motion for the bulk and brane fields are consistent with each other. We have

$$
\begin{aligned}
\frac{\delta \mathcal{L}}{\delta \mathbf{V}_{\mathbf{2}}} & \equiv \frac{1}{4} D \bar{D}^{2} D \mathbf{V}_{\mathbf{2}}-2 \partial_{5}\left[\mathbf{Z}_{\mathbf{2}}-2 \mathbf{G} \delta(z)\right]=0 \\
\frac{\delta \mathcal{L}}{\delta \mathbf{V}} & \equiv \delta(z)\left\{-4\left[\mathbf{Z}_{\mathbf{2}}-2 \mathbf{G} \delta(z)\right] \frac{\delta \mathbf{G}}{\delta \mathbf{V}}+\frac{\delta \mathcal{L}_{4}}{\delta \mathbf{V}}\right\}=0,
\end{aligned}
$$

so that both equations require $\mathbf{Z}_{\mathbf{2}}$ to have the same singular part,

$$
\mathbf{Z}_{\mathbf{2}}=2 \mathbf{G} \delta(z)+\text { n.s. }
$$

where "n.s." stands for non-singular terms. As $\mathbf{Z}_{\mathbf{2}}=\partial_{5} \mathbf{V}_{\mathbf{2}}-\frac{1}{2}\left(\mathbf{\Phi}_{\mathbf{2}}+\mathbf{\Phi}_{\mathbf{2}}{ }^{\dagger}\right)$, the singular term can arise from a jump in the odd superfield $\mathbf{V}_{\mathbf{2}}$,

$$
\partial_{5} \mathbf{V}_{\mathbf{2}}=2 \delta(z) \mathbf{V}_{\mathbf{2}}^{(+)}+\text {n.s. }
$$

or from the even superfield $\boldsymbol{\Phi}_{\mathbf{2}}$ having a singular part. If we write

$$
\mathbf{\Phi}_{2}=\widetilde{\mathbf{\Phi}}_{2}+4 \mathbf{K} \delta(z)
$$

with $\widetilde{\mathbf{\Phi}}_{2}$ being non-singular, we find that eq. (3.16) gives rise to a boundary condition

$$
\mathbf{V}_{\mathbf{2}} \stackrel{+0}{=} \mathbf{J}=\mathbf{K}+\mathbf{K}^{\dagger}+\mathbf{G}
$$

which coincides exactly with the boundary condition found in the boundary picture. Moreover, the gauge transformation of $\mathbf{\Phi}_{\mathbf{2}}$, eq. (2.14), when split into the singular and non-singular parts, gives

$$
\delta_{u} \mathbf{K}=\boldsymbol{\Lambda}_{\mathbf{2}}^{(+)}(u), \quad \delta_{u} \widetilde{\boldsymbol{\Phi}}_{\mathbf{2}}=2 \partial_{5} \boldsymbol{\Lambda}_{\mathbf{2}}(u)-4 \boldsymbol{\Lambda}_{\mathbf{2}}^{(+)}(u) \delta(z),
$$

which implies that the gauge and supersymmetry transformations of $\mathbf{K}$ are exactly as in eq. (3.10). We conclude, therefore, that the boundary compensator $\mathbf{K}$ corresponds to the singular part of $\boldsymbol{\Phi}_{\mathbf{2}}$ in the orbifold picture.

\subsection{OP with non-singular $\Phi_{2}$}

There is another way to approach bulk-brane coupling in the orbifold picture. Let us require that $\boldsymbol{\Phi}_{\mathbf{2}}$ be non-singular. This forces us to modify gauge and supersymmetry transformations of $\boldsymbol{\Phi}_{\mathbf{2}}$ in a way that makes them non-singular, which gives

$$
\begin{aligned}
& \delta_{u}^{\prime} \boldsymbol{\Phi}_{\mathbf{2}}=2 \partial_{5} \boldsymbol{\Lambda}_{\mathbf{2}}(u)-4 \boldsymbol{\Lambda}_{\mathbf{2}}^{(+)}(u) \delta(z) \\
& \delta_{\eta}^{\prime} \mathbf{\Phi}_{\mathbf{2}}=(\eta Q+\bar{\eta} \bar{Q}) \mathbf{\Phi}_{\mathbf{2}}+2 \partial_{5} \boldsymbol{\Lambda}_{\mathbf{2}}(\eta)-4 \boldsymbol{\Lambda}_{\mathbf{2}}^{(+)}(\eta) \delta(z) .
\end{aligned}
$$


With this modification, $\mathbf{Z}_{\mathbf{2}}$ is no longer gauge invariant

$$
\delta_{u}^{\prime} \mathbf{Z}_{\mathbf{2}}=2\left[\boldsymbol{\Lambda}_{\mathbf{2}}{ }^{(+)}(u)+\left(\boldsymbol{\Lambda}_{\mathbf{2}}{ }^{(+)}(u)\right)^{\dagger}\right] \delta(z) .
$$

Therefore, the right bulk-plus-brane Lagrangian now is

$$
\mathcal{L}=\frac{1}{8} \mathbf{V}_{\mathbf{2}} D \bar{D}^{2} D \mathbf{V}_{\mathbf{2}}+\left[\mathbf{Z}_{\mathbf{2}}-2 \mathbf{J} \delta(z)\right]^{2}+\mathcal{L}_{4} \delta(z)
$$

where $\mathbf{J}$ is required to transform as in eq. (3.9) in order for $\mathcal{L}$ to be supersymmetric. As in the boundary picture, we are lead to $\mathbf{J}$ of the form (3.11), explicitly containing the compensator K. Note that, unlike the boundary picture case, we can make a replacement

$$
\left.\boldsymbol{\Lambda}_{\mathbf{2}}{ }^{(+)}(\eta) \longrightarrow \boldsymbol{\Lambda}_{\mathbf{J}}(\eta) \equiv \boldsymbol{\Lambda}_{\mathbf{2}}{ }^{(+)}(\eta)\right|_{\mathbf{V}_{\mathbf{2}}=\mathbf{J}}
$$

in the supersymmetry transformations of $\mathbf{J}, \mathbf{K}$, and $\mathbf{\Phi}_{\mathbf{2}}$, and the Lagrangian (3.23) would still be supersymmetric without using boundary conditions.

The two orbifold picture constructions are, obviously, related by the field redefinition (3.18). The advantage of the formulation with a singular $\boldsymbol{\Phi}_{2}$ is that it avoids explicit appearance of the compensator $\mathbf{K}$. We will see more explicitly how the two approaches are related when we consider the component formulation.

\section{Bulk-brane coupling in components}

In this section, we show how to go from the superfield bulk-brane coupling established in the previous section, to its component form. In the boundary picture, we find that the $Y$-term of ref. [6] arises naturally from the extra superfield boundary term in $S_{5}^{\prime \prime}$. In the orbifold picture, we find that in order to arrive at the form of the coupling established for the Horava-Witten and Randall-Sundrum scenarios, one has to do a partial field redefinition.

\subsection{Boundary conditions}

In both the boundary and orbifold picture, the boundary condition is given by eq. (3.19). As $\mathbf{J}$ is a real vector superfield, we write its components as follows (see Appendix A)

$$
\mathbf{J}=\left(C_{J}, \chi_{J}, M_{J} ; J_{m}, \lambda_{J}, D_{J}\right) .
$$

With $\mathbf{V}_{\mathbf{2}}$ being in the WZ gauge and given by eq. (2.10), the boundary condition (3.19) splits into two sets of component boundary conditions. The first set requires the three lowest components of $\mathbf{J}$ to vanish:

$$
C_{J}=\chi_{J}=M_{J}=0 .
$$

The second set gives the actual boundary conditions in the component formulation,

$$
A_{m} \stackrel{+0}{=} J_{m}, \quad \lambda_{1} \stackrel{+0}{=} \lambda_{J}, \quad X_{3}-\partial_{5} \Phi \stackrel{+0}{=} D_{J}
$$




\subsection{Compensator (super)field}

The set of restrictions on $\mathbf{J}$, given in eq. (4.2), fixes $\mathbf{K}$ up to a single real field $K$. To see how this happens, we first define the components of $\mathbf{G}$ and $\mathbf{K}$ in a general way

$$
\mathbf{G}=\left(C_{G}, \chi_{G}, M_{G} ; G_{m}, \lambda_{G}, D_{G}\right), \quad \mathbf{K}=\left(\phi_{K}, \psi_{K}, F_{K}\right) .
$$

Writing $\mathbf{J}=\mathbf{K}+\mathbf{K}^{\dagger}+\mathbf{G}$ in components, we find

$$
\begin{gathered}
C_{J}=\phi_{K}+\phi_{K}^{*}+C_{G}, \quad \chi_{J}=-i \sqrt{2} \psi_{K}+\chi_{G}, \quad M_{J}=-2 i F_{K}+M_{G} \\
J_{m}=-i \partial_{m}\left(\phi_{K}-\phi_{K}^{*}\right)+G_{m}, \quad \lambda_{J}=\lambda_{G}, \quad D_{J}=D_{G} .
\end{gathered}
$$

The restriction (4.2) now gives three equations on the components of $\mathbf{K}$, which leave undetermined only the imaginary part of its lowest component. Denoting the latter by $K$, we have

$$
\mathbf{K}=\left(-\frac{1}{2} C_{G}+\frac{i}{2} K, \quad-\frac{i}{\sqrt{2}} \chi_{G}, \quad-\frac{i}{2} M_{G}\right) .
$$

With this definition of $K$, the non-zero components of $\mathbf{J}$ become

$$
J_{m}=G_{m}+\partial_{m} K, \quad \lambda_{J}=\lambda_{G}, \quad D_{J}=D_{G}
$$

Gauge and supersymmetry transformations of the components of $\mathbf{K}$ and $\mathbf{G}$ can be found from the superfield transformations given in eqs. (3.10) and (3.12), respectively. (For supersymmetry transformations, eq. (A.4) is useful.) We find, for example,

$$
\begin{aligned}
\delta_{u} \phi_{K} & =\frac{i}{2} u^{(+)}, \quad \delta_{\eta} \phi_{K}=\sqrt{2} \eta \psi_{K} \\
\delta_{u} C_{G} & =0, \quad \delta_{\eta} C_{G}=i \eta \chi_{G}+\text { h.c. }
\end{aligned}
$$

Applying these transformations to the lowest component of eq. (4.6), we obtain the following gauge and supersymmetry transformations of $K$,

$$
\delta_{u} K=u^{(+)}, \quad \delta_{\eta} K=-\eta \chi_{G}+\text { h.c. }
$$

Analogous treatment of the other two components in eq. (4.6) reproduces the boundary conditions (4.3) for $A_{m}$ and $\lambda_{1}$. Note that these boundary conditions would not arise here if we make the replacement (3.24) in the supersymmetry transformation of $\mathbf{K}$.

\subsection{Boundary picture}

The boundary picture action $S_{5}^{\prime \prime}$, eq. (3.5), appropriate for the odd $A_{m}$, differs from the original bulk action $S_{5}$ by a boundary term that we call $Y$-term [6, 11],

$$
S_{5}^{\prime \prime}=S_{5}+\int_{\partial \mathcal{M}} Y^{\prime \prime}
$$


This $Y^{\prime \prime}$-term is a sum of the $Y^{\prime}$-term for the action $S_{5}^{\prime}$, eq. (2.20), and of the boundary superfield term in eq. (3.5),

$$
Y^{\prime \prime}=Y^{\prime}+2\left(\mathbf{Z}_{\mathbf{2}} \mathbf{V}_{\mathbf{2}}\right)_{\left.\right|_{\theta^{2}} \bar{\theta}^{2}}=F_{m 5} A^{m}-\frac{1}{2}\left(\lambda_{1} \lambda_{2}+\text { h.c. }\right) .
$$

This way we reproduce the $Y$-term of the form suggested in ref. [6], with the $F_{m 5} A^{m}$ term present. For the total bulk-plus-boundary action (3.7), we find

$$
\begin{aligned}
S= & S_{5}+\int_{\partial \mathcal{M}}\left[F_{m 5} A^{m}-\frac{1}{2}\left(\lambda_{1} \lambda_{2}+\text { h.c. }\right)\right] \\
& +\frac{1}{2} \int_{\partial \mathcal{M}}\left[\mathcal{L}_{4}+2 \Phi D_{J}+2\left(\lambda_{2} \lambda_{J}+\text { h.c. }\right)-2 F_{m 5} J^{m}\right] .
\end{aligned}
$$

As we will show in Section 5.2, this action is supersymmetric under the bulk supersymmetry transformations (2.8) and appropriate transformations of the components of $\mathbf{J}$. We will find, however, that showing this requires using the boundary condition (4.3) for $A_{m}$ (and also the one for $\lambda_{1}$, unless we eliminate auxiliary fields).

We can simplify the form of the action by explicitly using some or all of the boundary conditions (4.3). Using the one for $A_{m}$, we obtain

$$
\begin{aligned}
S_{1}= & S_{5}+\int_{\partial \mathcal{M}}\left[-\frac{1}{2}\left(\lambda_{1} \lambda_{2}+\text { h.c. }\right)\right] \\
& +\frac{1}{2} \int_{\partial \mathcal{M}}\left[\mathcal{L}_{4}+2 \Phi D_{J}+2\left(\lambda_{2} \lambda_{J}+\text { h.c. }\right)\right] .
\end{aligned}
$$

Using the boundary conditions for both $A_{m}$ and $\lambda_{1}$, we get

$$
S_{2}=S_{5}+\frac{1}{2} \int_{\partial \mathcal{M}}\left[\mathcal{L}_{4}+2 \Phi D_{J}+\left(\lambda_{2} \lambda_{J}+\text { h.c. }\right)\right] .
$$

We will find that supersymmetry of $S_{1}$ depends on using the boundary conditions for $A_{m}$ and $\lambda_{1}$, whereas $S_{2}$ is supersymmetric provided the third boundary condition in eq. (4.3) is also used. The reason for this is explained in Appendix C.

\subsection{OP with singular fields}

In the orbifold picture, all $\delta(z)$-dependent terms in the bulk-plus-brane Lagrangian (3.14) come from the following part

$$
\begin{aligned}
{\left[\mathbf{Z}_{2}-\right.} & 2 \mathbf{G} \delta(z)]_{\mid \theta^{2} \bar{\theta}^{2}}^{2}= \\
- & {\left[\lambda_{2}-2 \chi_{G} \delta(z)\right]\left[\partial_{5} \lambda_{1}-2 \lambda_{G} \delta(z)+\frac{i}{2} \sigma^{m} \partial_{m}\left[\bar{\lambda}_{2}-2 \bar{\chi}_{G} \delta(z)\right]\right]+h . c . } \\
- & {\left[\Phi+2 C_{G} \delta(z)\right]\left[\partial_{5}\left(X_{3}-\partial_{5} \Phi\right)-2 D_{G} \delta(z)-\frac{1}{2} \partial_{m} \partial^{m}\left[\Phi+2 C_{G} \delta(z)\right]\right] } \\
& -\frac{1}{2}\left[F_{m 5}+2 G_{m} \delta(z)\right]^{2}+\frac{1}{2}\left[X_{12}-2 i M_{G} \delta(z)\right]\left[X_{12}^{*}+2 i M_{G}^{*} \delta(z)\right] .
\end{aligned}
$$


Dropping some total $\partial_{5}$ derivatives, irrelevant in the orbifold picture, the total Lagrangian can be brought to the following form

$$
\mathcal{L}=\mathcal{L}_{5}+\left[\mathcal{L}_{4}+B_{1}\right] \delta(z)+B_{2} \delta(z)^{2}+B_{3} \delta^{\prime}(z)
$$

where

$$
\begin{aligned}
B_{1}= & 2 \lambda_{2} \lambda_{G}+2 i \chi_{G} \sigma^{m} \partial_{m} \bar{\lambda}_{2}-i X_{12}^{*} M_{G}+\text { h.c. } \\
& -2 F_{m 5} G^{m}+2 \Phi D_{G}+2 C_{G} \partial_{m} \partial^{m} \Phi \\
B_{2}= & -4 \chi_{G} \lambda_{G}-2 i \chi_{G} \sigma^{m} \partial_{m} \bar{\chi}_{G}+\text { h.c. } \\
& -2 G_{m} G^{m}+2 C_{G} \partial_{m} \partial^{m} C_{G}+4 C_{G} D_{G}+2 M_{G} M_{G}^{*} \\
B_{3}= & -2 \chi_{G} \lambda_{1}+\text { h.c. }+2 C_{G}\left(X_{3}-\partial_{5} \Phi\right) .
\end{aligned}
$$

This Lagrangian, by construction, is supersymmetric under the original supersymmetry transformations (2.8) of the bulk fields. However, its $\delta(z)$-dependent terms happen to be more complicated than those in the (more complicated) supergravity theories. We will see next that this apparent paradox can be resolved by a simple field redefinition.

\subsection{OP with singular $A_{5}$}

From eq. (3.16), we know that $\mathbf{Z}_{\mathbf{2}}-2 \mathbf{G} \delta(z)$ is non-singular. Using the component forms of $\mathbf{Z}_{2}$ and $\mathbf{G}$, eqs. (2.17) and (4.4), respectively, we find that the following fields,

$$
\begin{aligned}
\widetilde{\Phi} & \equiv \Phi+2 C_{G} \delta(z) \\
\widetilde{\lambda}_{2} & \equiv \lambda_{2}-2 \chi_{G} \delta(z) \\
\widetilde{X}_{12} & \equiv X_{12}-2 i M_{G} \delta(z) \\
\widetilde{X}_{3} & \equiv X_{3}+2 C_{G} \delta^{\prime}(z),
\end{aligned}
$$

are non-singular. ${ }^{8}$ A glance at eq. (4.15) shows that transforming to the new fields absorbs most of the $\delta(z)$ terms. Performing the field redefinition, and omitting the tildes, we find

$$
\mathcal{L}=\mathcal{L}_{5}^{(\mathcal{F})}+\left[\mathcal{L}_{4}+2 \Phi D_{G}+2\left(\lambda_{2} \lambda_{G}+\text { h.c. }\right)\right] \delta(z)
$$

where $\mathcal{L}_{5}^{(\mathcal{F})}$ is obtained from the original Lagrangian $\mathcal{L}_{5}$, eq. (2.6), by replacing $F_{m 5}$ with

$$
\mathcal{F}_{m 5}=F_{m 5}+2 G_{m} \delta(z) .
$$

\footnotetext{
${ }^{8}$ When we say that a field is non-singular, we mean that it is non-singular when equations of motion are used. Note that we reserve the word "on-shell" to mean "when auxiliary fields are eliminated."
} 
Performing the redefinition (4.18) in the supersymmetry transformations (2.8) requires using the transformations of the components of $\mathbf{G}$. Since $\mathbf{G}$ transforms as in eq. (3.12), its components transform according to eq. (A.4). After a short calculation, we find the following modified supersymmetry transformations of the bulk fields,

$$
\begin{aligned}
\delta_{\eta} A_{m} & =i \eta \sigma_{m} \bar{\lambda}_{1}+h . c . \\
\delta_{\eta} A_{5} & =-\eta \lambda_{2}-2 \eta \chi_{G} \delta(z)+h . c . \\
\delta_{\eta} \Phi & =-i \eta \lambda_{2}+h . c . \\
\delta_{\eta} \lambda_{1} & =\sigma^{m n} \eta F_{m n}+i\left(X_{3}-\partial_{5} \Phi\right) \eta \\
\delta_{\eta} \lambda_{2} & =-i \sigma^{m} \bar{\eta}\left[F_{m 5}+2 G_{m} \delta(z)\right]-\sigma^{m} \bar{\eta} \partial_{m} \Phi-i X_{12} \eta \\
\delta_{\eta} X_{12} & =2 i \bar{\eta}\left[\partial_{5} \bar{\lambda}_{1}-2 \lambda_{G} \delta(z)\right]-2 \overline{\eta \sigma}^{m} \partial_{m} \lambda_{2} \\
\delta_{\eta} X_{3} & =-i \eta \partial_{5} \lambda_{2}-\eta \sigma^{m} \partial_{m} \bar{\lambda}_{1}+h . c .
\end{aligned}
$$

The modifications can be summarized as follows: 1) replace $F_{m 5}$ with $\mathcal{F}_{m 5}, 2$ ) modify the transformation of $A_{5}$ by adding the following singular piece

$$
\delta_{\eta}^{(\mathrm{s})} A_{5}=-2\left(\eta \chi_{G}+\text { h.c. }\right) \delta(z)
$$

and 3) modify the transformation of $X_{12}$ (the even auxiliary field) by terms that make it nonsingular when the boundary conditions (4.3) are used. When auxiliary fields are eliminated, we need only the first two prescriptions. Therefore, in the on-shell formulation, we match the supergravity bulk-brane coupling construction of ref. [7].

Note that after the redefinition (4.18), we still have one singular field left: $A_{5}$. From eq. (3.16) and the boundary conditions (4.3), we have

$$
F_{m 5}+2 G_{m} \delta(z)=n . s ., \quad A_{m} \stackrel{+0}{=} G_{m}+\partial_{m} K \Rightarrow A_{5}=2 K \delta(z)+n . s .
$$

We see that the singular part of $A_{5}$ is directly related to the compensator field $K$. If we redefine $A_{5}$ to make it non-singular, we find that its supersymmetry transformation also becomes non-singular:

$$
\widetilde{A}_{5}=A_{5}-2 K \delta(z) \quad \Rightarrow \quad \delta_{\eta} \widetilde{A}_{5}=-\eta \lambda_{2}+h . c .
$$

If we now replace $A_{5}$ with $\widetilde{A}_{5}$ in the expression for $\mathcal{F}_{m 5}$, eq. $(4.20)$, we find that $G_{m}$ gets replaced by $J_{m}=G_{m}+\partial_{m} K$ :

$$
\mathcal{F}_{m 5}=F_{m 5}+2 G_{m} \delta(z)=\widetilde{F}_{m 5}+2 J_{m} \delta(z)
$$

As we will see next, after this final field redefinition we come exactly to the construction in which the superfield $\mathbf{\Phi}_{\mathbf{2}}$ is non-singular from the start. 


\subsection{OP with non-singular fields}

In the case with non-singular $\mathbf{\Phi}_{\mathbf{2}}$, the bulk-plus-brane Lagrangian is given by eq. (3.23). As the lowest components of $\mathbf{J}$ (unlike $\mathbf{G}$ ) vanish, $C_{J}=\chi_{J}=M_{J}=0$, the component form of the Lagrangian is simple without any field redefinitions:

$$
\mathcal{L}=\mathcal{L}_{5}^{(\mathcal{F})}+\left[\mathcal{L}_{4}+2 \Phi D_{J}+2\left(\lambda_{2} \lambda_{J}+\text { h.c. }\right)\right] \delta(z) .
$$

As before, we must replace $F_{m 5}$ by $\mathcal{F}_{m 5}$ that is now given by

$$
\mathcal{F}_{m 5}=F_{m 5}+2 J_{m} \delta(z) \text {. }
$$

Superfield supersymmetry transformations are now different from those in eq. (2.11). They are modified as in eq. (3.21) so that the transformation of $\boldsymbol{\Phi}_{\mathbf{2}}$ is non-singular. We should, however, make the choice: whether or not to make the replacement (3.24). Because of the last statement in Section 4.2, the component Lagrangian will be supersymmetric without using boundary conditions provided we do make the replacement (3.24). The component supersymmetry transformations then become

$$
\begin{aligned}
\delta_{\eta} A_{m} & =i \eta \sigma_{m} \bar{\lambda}_{1}+h . c . \\
\delta_{\eta} A_{5} & =-\eta \lambda_{2}+h . c . \\
\delta_{\eta} \Phi & =-i \eta \lambda_{2}+h . c . \\
\delta_{\eta} \lambda_{1} & =\sigma^{m n} \eta F_{m n}+i\left(X_{3}-\partial_{5} \Phi\right) \eta \\
\delta_{\eta} \lambda_{2} & =-i \sigma^{m} \bar{\eta}\left[F_{m 5}+2 J_{m} \delta(z)\right]-\sigma^{m} \bar{\eta} \partial_{m} \Phi-i X_{12} \eta \\
\delta_{\eta} X_{12} & =2 i \bar{\eta}\left[\partial_{5} \bar{\lambda}_{1}-2 \lambda_{J} \delta(z)\right]-2 \overline{\eta \sigma}^{m} \partial_{m} \lambda_{2} \\
\delta_{\eta} X_{3} & =-i \eta \partial_{5} \lambda_{2}-\eta \sigma^{m} \partial_{m} \bar{\lambda}_{1}+h . c .
\end{aligned}
$$

This differs from the original transformations (2.8) by $\delta(z)$-dependent modifications that are now all covered by one simple rule [5, 6, 11]: the modifications must make the transformations non-singular when the boundary conditions are used.

We conclude that there are two alternative simple forms of the bulk-brane coupling in the orbifold picture: one with the compensator $K$ appearing explicitly via $J_{m}$, and the other where the role of the compensator is played by the singular part of $A_{5}$. The two formulations are related to each other by the redefinition (4.24) of $A_{5}$.

\section{On-shell coupling}

In this section, we go on-shell (eliminate auxiliary fields) and check explicitly that the bulkplus-brane/boundary actions we constructed are indeed supersymmetric. We find that some boundary conditions have to be used for supersymmetry in the boundary picture. At the end of the section, we give an explicit example of a coupled bulk-brane system which makes contact with the supergravity construction of ref. [7]. 


\subsection{Modified Bianchi identity}

As we established, in the orbifold picture, a part of the bulk-brane coupling prescription consists in replacing $F_{m 5}$ with $\mathcal{F}_{m 5}$ both in the Lagrangian and in the supersymmetry transformations. Let us now generalize this to the following shift

$$
F_{M N} \equiv \partial_{M} A_{N}-\partial_{N} A_{M} \quad \longrightarrow \quad \mathcal{F}_{M N}=F_{M N}+B_{M N}
$$

On-shell $\left(X_{a}=0\right)$ and after the shift, the bulk Lagrangian (2.1) turns into

$$
\mathcal{L}_{5}^{(\mathcal{F})}=-\frac{1}{4} \mathcal{F}_{M N} \mathcal{F}^{M N}-\frac{1}{2} \partial_{M} \Phi \partial^{M} \Phi-\frac{i}{2} \widetilde{\Lambda}^{i} \Gamma^{M} \partial_{M} \Lambda_{i}
$$

and the corresponding supersymmetry transformations become

$$
\begin{aligned}
\delta_{\mathcal{H}} A_{M} & =i \widetilde{\mathcal{H}}^{i} \Gamma_{M} \Lambda_{i} \\
\delta_{\mathcal{H}} \Phi & =i \widetilde{\mathcal{H}}^{i} \Lambda_{i} \\
\delta_{\mathcal{H}} \Lambda_{i} & =\left(\Sigma^{M N} \mathcal{F}_{M N}+\Gamma^{M} \partial_{M} \Phi\right) \mathcal{H}_{i} .
\end{aligned}
$$

Supersymmetry transformation of the bulk Lagrangian now produces not only the total derivative, but also extra terms involving $B_{M N}$ :

$$
\begin{array}{r}
\delta_{\mathcal{H}} \mathcal{L}_{5}=\partial_{M} \widetilde{K}^{M}-\frac{1}{2} \mathcal{F}^{M N} \delta_{\mathcal{H}} B_{M N}-\frac{i}{2} \widetilde{\eta}^{i} \Gamma^{M N K} \Lambda_{i} \partial_{K} \mathcal{F}_{M N} \\
\widetilde{K}^{M}=-\mathcal{F}^{M N} \delta_{\mathcal{H}} A_{N}-\delta_{\mathcal{H}} \Phi \partial^{M} \Phi+\frac{i}{2} \widetilde{\Lambda}^{i} \Gamma^{M} \delta_{\mathcal{H}} \Lambda_{i} .
\end{array}
$$

The last term in $\delta_{\mathcal{H}} \mathcal{L}_{5}$ is the famous contribution due to the "modified Bianchi identity." Note that in the boundary picture, we have $B_{M N}=0$ and the total derivative term is important; in the orbifold picture, $B_{M N} \neq 0$ and the total derivative is irrelevant.

\subsection{Boundary picture}

The bulk-plus-boundary action in the boundary picture is given by eq. (3.7),

$$
\begin{aligned}
S= & S_{5}+\int_{\partial \mathcal{M}}\left[F_{m 5} A^{m}-\frac{1}{2}\left(\lambda_{1} \lambda_{2}+\text { h.c. }\right)\right] \\
& +\frac{1}{2} \int_{\partial \mathcal{M}}\left[\mathcal{L}_{4}+2 \Phi D_{J}+2\left(\lambda_{2} \lambda_{J}+\text { h.c. }\right)-2 F_{m 5} J^{m}\right] .
\end{aligned}
$$

Supersymmetry variation of $S_{5}$ produces the following boundary term,

$$
\delta_{\eta} S_{5}=\int_{\partial \mathcal{M}}\left(-\widetilde{K}^{5}\right)=\int_{\partial \mathcal{M}}\left[-F^{m 5} \delta_{\eta} A_{m}+\delta_{\eta} \Phi \partial_{5} \Phi-\frac{1}{2}\left(\lambda_{2} \delta_{\eta} \lambda_{1}-\lambda_{1} \delta_{\eta} \lambda_{2}+\text { h.c. }\right)\right] .
$$

To find the variation of the total action, we need to know supersymmetry transformations of $J_{m}, \lambda_{J}$, and $D_{J}$. We know that components of $\mathbf{G}$ transform as in eq. (A.4), so that, in 
particular, ${ }^{9}$

$$
\begin{aligned}
\delta_{\eta} G_{m} & =i \eta \sigma_{m} \bar{\lambda}_{G}+\partial_{m}\left(\eta \chi_{G}\right)+\text { h.c. } \\
\delta_{\eta} \lambda_{G} & =\sigma^{m n} \eta G_{m n}+i \eta D_{G} \\
\delta_{\eta} D_{G} & =\overline{\eta \sigma}^{m} \partial_{m} \lambda_{G}+\text { h.c. }
\end{aligned}
$$

From eq. (4.7) and the supersymmetry transformation (4.9) of $K$, it then follows that

$$
\begin{aligned}
\delta_{\eta} J_{m} & =i \eta \sigma_{m} \bar{\lambda}_{J}+h . c . \\
\delta_{\eta} \lambda_{J} & =\sigma^{m n} \eta J_{m n}+i \eta D_{J} \\
\delta_{\eta} D_{J} & =\overline{\eta \sigma}^{m} \partial_{m} \lambda_{J}+\text { h.c. }
\end{aligned}
$$

Using these transformations together with the ones for the bulk fields, eq. (2.8), we find

$$
\delta_{\eta} S=\int_{\partial \mathcal{M}}\left[\eta \sigma^{m n} \lambda_{2}\left(F_{m n}-J_{m n}\right)+\text { h.c. }+\left(A^{m}-J^{m}\right) \delta_{\eta} F_{m 5}\right] .
$$

For the action (4.13), obtained from $S$ by using the $A_{m}$ boundary condition, we have

$$
\delta_{\eta} S_{1}=\int_{\partial \mathcal{M}}\left[\eta \sigma^{m n} \lambda_{2}\left(F_{m n}-J_{m n}\right)-i \eta \sigma^{m}\left(\bar{\lambda}_{1}-\bar{\lambda}_{J}\right) F_{m 5}+\text { h.c. }\right] .
$$

For the action (4.14), obtained from $S_{1}$ by using the $\lambda_{1}$ boundary condition, we get

$$
\begin{aligned}
\delta_{\eta} S_{2}=\int_{\partial \mathcal{M}}[ & {\left[\frac{1}{2} \eta \sigma^{m n} \lambda_{2}\left(F_{m n}-J_{m n}\right)-\frac{i}{2} \eta \sigma^{m}\left(\bar{\lambda}_{1}-\bar{\lambda}_{J}\right) F_{m 5}\right.} \\
& \left.\quad-\frac{i}{2} \eta \lambda_{2}\left(\partial_{5} \Phi+D_{J}\right)-\frac{1}{2} \eta \sigma^{m}\left(\bar{\lambda}_{1}-\bar{\lambda}_{J}\right) \partial_{m} \Phi+\text { h.c. }\right] .
\end{aligned}
$$

We conclude that each action is supersymmetric, and in each case supersymmetry of the action depends on using some boundary conditions. The basic pattern we observe is: the more boundary conditions are used to simplify the action, the more of them are needed to prove its supersymmetry. The way to predict which boundary conditions are needed in each case is given in Appendix C.

\subsection{Orbifold picture}

In the orbifold picture, with singular $A_{5}$, we have

$$
\begin{gathered}
B_{m n}=0, \quad B_{m 5}=-B_{5 m}=2 G_{m} \delta(z) \\
\mathcal{F}_{m n}=F_{m n}, \quad \mathcal{F}_{m 5}=F_{m 5}+2 G_{m} \delta(z) .
\end{gathered}
$$

The bulk-plus-brane Lagrangian is given by eq. (4.19),

$$
\mathcal{L}=\mathcal{L}_{5}^{(\mathcal{F})}+\mathcal{L}_{4}^{\prime} \delta(z), \quad \mathcal{L}_{4}^{\prime}=\mathcal{L}_{4}+2 \Phi D_{G}+2\left(\lambda_{2} \lambda_{G}+\text { h.c. }\right) .
$$

\footnotetext{
${ }^{9}$ In our notation, $v_{m n}=\partial_{m} v_{n}-\partial_{n} v_{m}, G_{m n}=\partial_{m} G_{n}-\partial_{n} G_{m}, J_{m n}=\partial_{m} J_{n}-\partial_{n} J_{m}$.
} 
Supersymmetry variation of $\mathcal{L}_{5}^{(\mathcal{F})}$ gives

$$
\delta_{\eta} \mathcal{L}_{5}^{(\mathcal{F})}=\left\{2\left(\eta \sigma^{m n} \lambda_{2}+\text { h.c. }\right) G_{m n}-\mathcal{F}^{m 5}\left[2 \delta_{\eta} G_{m}+\partial_{m}\left(\widetilde{\delta}_{\eta}^{(s)} A_{5}\right)\right]\right\} \delta(z),
$$

where the terms with $G_{m}$ follow from the $B_{M N}$ terms in eq. (5.4), and the last term follows from the modification (4.22) in the supersymmetry transformation of $A_{5}$ with

$$
\widetilde{\delta}_{\eta}^{(s)} A_{5} \equiv-2 \eta \chi_{G}+\text { h.c. }=2 \delta_{\eta} K .
$$

Note that the sum of the terms in the square bracket gives $2 \delta_{\eta} J_{m}$. For $\mathcal{L}_{4}^{\prime}$, we find

$$
\delta_{\eta}\left(\lambda_{2} \lambda_{G}+\text { h.c. }+\Phi D_{G}\right)=-\eta \sigma^{m n} \lambda_{2} G_{m n}+i \eta \sigma^{m} \bar{\lambda}_{G} \mathcal{F}_{m 5}+\text { h.c. }
$$

from which we conclude that the total Lagrangian $\mathcal{L}$ is supersymmetric, $\delta_{\eta} \mathcal{L}=0$, without using any boundary conditions.

\subsection{Example}

To make contact with the supergravity construction of ref. [7], we consider an example with one brane-localized chiral superfield $\boldsymbol{\Phi}$ and

$$
\mathbf{G}=\boldsymbol{\Phi}^{\dagger} \boldsymbol{\Phi}
$$

With $\mathbf{\Phi}=(\phi, \psi, F)$, the components of $\mathbf{G}$ are given by

$$
\begin{aligned}
C_{G} & =\phi \phi^{*} \\
\chi_{G} & =-i \sqrt{2} \phi^{*} \psi \\
M_{G} & =-2 i F \phi^{*} \\
G_{m} & =i\left(\phi \partial_{m} \phi^{*}-\phi^{*} \partial_{m} \phi\right)+\psi \sigma_{m} \bar{\psi} \\
\lambda_{G} & =\sqrt{2} \sigma^{m} \bar{\psi} \partial_{m} \phi+i \sqrt{2} \psi F^{*} \\
D_{G} & =2 F F^{*}-2 \partial_{m} \phi \partial^{m} \phi^{*}-\left(i \psi \sigma^{m} \partial_{m} \bar{\psi}+\text { h.c. }\right) .
\end{aligned}
$$

The supersymmetry transformation of the compensator $K$, eq. (4.9), can now be written as follows, 10

$$
\delta_{\eta} K=i \sqrt{2} \phi^{*} \eta \psi+h . c .=i\left(\phi^{*} \delta_{\eta} \phi-\phi \delta_{\eta} \phi^{*}\right)
$$

which clearly shows that we cannot "gauge fix" the compensator by making it a function of the matter fields. To complete the setup, we choose

$$
\mathcal{L}_{4}=\int d^{2} \theta d^{2} \bar{\theta} \Phi^{\dagger} \mathbf{\Phi}=F F^{*}-\partial_{m} \phi \partial^{m} \phi^{*}-\left(\frac{i}{2} \psi \sigma^{m} \partial_{m} \bar{\psi}+\text { h.c. }\right) .
$$

\footnotetext{
${ }^{10}$ Note that this form of $\delta_{\eta} K$ implies $\delta_{\eta}^{(s)} A_{5}=2 i\left(\phi^{*} \delta_{\eta} \phi-\phi \delta_{\eta} \phi^{*}\right) \delta(z)$, which is remarkably similar to $\widetilde{\delta} C_{11 A B}$ in eq. (2.16) of the first paper in ref. [1].
} 
Plugging all the pieces into the bulk-plus-brane Lagrangian (5.13), and eliminating the auxiliary field $F$ by its equation of motion,

$$
F=-2 i \sqrt{2}(1+4 \Phi)^{-1} \lambda_{2} \psi
$$

we find that the on-shell Lagrangian is given by $\mathcal{L}=\mathcal{L}_{5}^{(\mathcal{F})}+\mathcal{L}_{4}^{\prime} \delta(z)$ with

$$
\begin{aligned}
\mathcal{L}_{4}^{\prime}= & (1+4 \Phi)\left[-\partial_{m} \phi \partial^{m} \phi^{*}-\left(\frac{i}{2} \psi \sigma^{m} \partial_{m} \bar{\psi}+\text { h.c. }\right)\right] \\
& +2 \sqrt{2}\left(\lambda_{2} \sigma^{m} \bar{\psi} \partial_{m} \phi+\text { h.c. }\right)-8(1+4 \Phi)^{-1}\left(\lambda_{2} \psi\right)\left(\bar{\lambda}_{2} \bar{\psi}\right) .
\end{aligned}
$$

The bulk Lagrangian $\mathcal{L}_{5}^{(\mathcal{F})}$ is obtained from $\mathcal{L}_{5}$ by replacing $F_{m 5}$ with

$$
\mathcal{F}_{m 5}=F_{m 5}+2 G_{m} \delta(z), \quad G_{m}=i\left(\phi \partial_{m} \phi^{*}-\phi^{*} \partial_{m} \phi\right)+\psi \sigma_{m} \bar{\psi}
$$

The same substitution must be made in the supersymmetry transformations (2.8), and, in addition, the transformation of $A_{5}$ should be modified by adding

$$
\delta^{(s)} A_{5}=2\left(\delta_{\eta} K\right) \delta(z)=2 i \sqrt{2} \phi^{*} \eta \psi \delta(z)+\text { h.c. }
$$

With these modifications, the total Lagrangian $\mathcal{L}$ is supersymmetric without using any boundary conditions.

We observe that the whole construction is identical to the one in supergravity [7]. It is also amusing to note that the brane Lagrangian (5.22) appears to be very similar to the one in supergravity: $(1+4 \Phi)$ plays the role of the induced metric, $\lambda_{2}$ is the "gravitino," $\lambda_{2} \sigma^{m} \bar{\psi} \partial_{m} \phi$ is the "Noether coupling" term, and $\left(\lambda_{2} \psi\right)\left(\bar{\lambda}_{2} \bar{\psi}\right)$ represents 4 -Fermi terms.

\section{Summary and Conclusions}

In this paper, we showed that the basic features of the supergravity bulk-brane coupling, present both in the Horava-Witten (11D) and Randall-Sundrum (5D) scenarios, appear also in the simplified globally supersymmetric model we considered (Mirabelli-Peskin model with odd $\left.A_{m}\right)$. Using the $4 \mathrm{D} N=1$ superfield formulation of the model, we showed that the full square structure [1] of the coupling in the orbifold picture is present already on the superfield level (see eq. (3.14)). In transition to the component formulation, one has to make some field redefinitions (see eq. (4.18)) in order to arrive at the established form of the coupling. After the redefinition, the full square structure remains only for the $\left(F_{m 5}+2 G_{m} \delta(z)\right)^{2}$ term in the Lagrangian (4.19). As the redefined fields are non-singular, the shift $F_{m 5} \rightarrow F_{m 5}+2 G_{m} \delta(z)$ in supersymmetry transformations is required to make the transformations non-singular. All together, we recover the "modified Bianchi identity" prescription for the coupling [1].

The only modification of the supersymmetry transformations, in the formulation of refs. [1, 7], that is not covered by the prescription "make them non-singular" [5, 6, 11] concerns the "orthogonal" component of the bulk gauge field. In fact, $A_{5}$ is the only field in this 
formulation which is singular. We showed that there is another formulation, where all fields are non-singular, and where the singular part of $A_{5}$ is replaced by a compensator field $K$. All modifications of the supersymmetry transformations are then covered by one simple rule.

Although optional in the orbifold picture, the presence of the compensator $K$ is unavoidable in the boundary picture construction. In both pictures, the boundary condition for the odd gauge field $A_{m}$ is $A_{m} \stackrel{+0}{=} J_{m}=G_{m}+\partial_{m} K$. The gauge transformation of $K$, given in eq. (4.9) (compare also with eq. (13.7) of ref. [6]), guarantees gauge invariance of the boundary condition. On the other hand, its supersymmetry transformation (4.9) is such that $K$ together with $C_{G}, \chi_{G}$, and $M_{G}$ (the lowest components of $\mathbf{G}$ ) combine into one chiral superfield (the compensator superfield $\mathbf{K}$, eq. (4.6)).

Our results shed some more light on the general structure of the supersymmetric bulkbrane coupling. They should also be useful in obtaining a more explicit (component) form of the coupling in the supersymmetric Randall-Sundrum scenario starting from the superfield formulation developed in ref. [12].

Acknowledgments. I would like to thank Hyun Min Lee and Christoph Ludeling for a discussion of an earlier version of this work.

\section{Appendix}

\section{A Superfield components}

Our supersymmetry conventions follow closely those of ref. [13]. For real vector and chiral superfields, we use the following shorthand notation,

$$
\mathbf{V}=\left(C, \chi, M ; v_{m}, \lambda, D\right), \quad \mathbf{\Phi}=(\phi, \psi, F)
$$

corresponding to the standard component expansions,

$$
\begin{aligned}
\mathbf{V}= & i \theta \chi+\frac{i}{2} \theta^{2} M-i \bar{\theta}^{2} \theta\left[\lambda+\frac{i}{2} \sigma^{m} \partial_{m} \bar{\chi}\right]+h . c . \\
& +C-\theta \sigma^{m} \bar{\theta} v_{m}+\frac{1}{2} \theta^{2} \bar{\theta}^{2}\left[D+\frac{1}{2} \partial_{m} \partial^{m} C\right] \\
& \mathbf{\Phi}=\left(1+i \theta \sigma^{m} \bar{\theta} \partial_{m}+\frac{1}{4} \theta^{2} \bar{\theta}^{2} \partial_{m} \partial^{m}\right) \phi+\sqrt{2}\left(\theta+\frac{i}{2} \theta^{2} \bar{\theta} \bar{\sigma}^{m} \partial_{m}\right) \psi+\theta^{2} F .
\end{aligned}
$$

When supersymmetry transformations have the standard form (without additional gauge transformations),

$$
\delta_{\eta} \mathbf{V}=(\eta Q+\bar{\eta} \bar{Q}) \mathbf{V}, \quad \delta_{\eta} \boldsymbol{\Phi}=(\eta Q+\bar{\eta} \bar{Q}) \mathbf{\Phi}
$$


the component transformations are as follows,

$$
\begin{aligned}
\delta_{\eta} C & =i \eta \chi+h . c . \\
\delta_{\eta} \chi & =\sigma^{m} \bar{\eta}\left(\partial_{m} C+i v_{m}\right)+\eta M \\
\delta_{\eta} M & =2 \bar{\eta}\left(\bar{\lambda}+i \bar{\sigma}^{m} \partial_{m} \chi\right) \\
\delta_{\eta} v_{m} & =i \eta \sigma_{m} \bar{\lambda}+\partial_{m}(\eta \chi)+h . c \\
\delta_{\eta} \lambda & =\sigma^{m n} \eta v_{m n}+i \eta D \\
\delta_{\eta} D & =\partial_{m}\left(\overline{\eta \sigma}^{m} \lambda+h . c .\right) \\
\delta_{\eta} \phi & =\sqrt{2} \eta \psi \\
\delta_{\eta} \psi & =i \sqrt{2} \sigma^{m} \bar{\eta} \partial_{m} \phi+\sqrt{2} \eta F \\
\delta_{\eta} F & =\partial_{m}\left(i \sqrt{2} \overline{\eta \sigma}^{m} \psi\right) .
\end{aligned}
$$

Components of gauge invariant superfields, $\mathbf{Z}_{\mathbf{2}}, \mathbf{W}_{\mathbf{2}}$, and $\mathbf{G}$, have exactly this form of supersymmetry transformations.

\section{B $\mathrm{V}_{2}$ and $\Phi_{2}$ without $\mathrm{WZ}$}

Our bulk superfields transform under supersymmetry according to eq. (2.11),

$$
\begin{aligned}
& \delta_{\eta} \mathbf{V}_{\mathbf{2}}=(\eta Q+\bar{\eta} \bar{Q}) \mathbf{V}_{\mathbf{2}}+\boldsymbol{\Lambda}_{\mathbf{2}}(\eta)+\boldsymbol{\Lambda}_{\mathbf{2}}(\eta)^{\dagger} \\
& \delta_{\eta} \mathbf{\Phi}_{\mathbf{2}}=(\eta Q+\bar{\eta} \bar{Q}) \mathbf{\Phi}_{\mathbf{2}}+2 \partial_{5} \boldsymbol{\Lambda}_{\mathbf{2}}(\eta) .
\end{aligned}
$$

Let us proceed without imposing the $\mathrm{WZ}$ gauge. If we take the gauge parameter in its general form, $\boldsymbol{\Lambda}_{\mathbf{2}}(\eta)=\left(a_{2}, \alpha_{2}, f_{2}\right)$, the component transformations become

$$
\begin{aligned}
\delta_{\eta} C_{2} & =i \eta \chi_{2}+a_{2}+h . c . \\
\delta_{\eta} \chi_{2} & =\sigma^{m} \bar{\eta}\left(\partial_{m} C_{2}+i v_{m}^{(2)}\right)+\eta M_{2}-i \sqrt{2} \alpha_{2} \\
\delta_{\eta} M_{2} & =2 \bar{\eta}\left(\bar{\lambda}_{(2)}+i \bar{\sigma}^{m} \partial_{m} \chi_{2}\right)-2 i f_{2} \\
\delta_{\eta} v_{m}^{(2)} & =i \eta \sigma_{m} \bar{\lambda}_{(2)}+\partial_{m}\left(\eta \chi_{2}-i a_{2}\right)+h . c \\
\delta_{\eta} \lambda_{(2)} & =\sigma^{m n} \eta v_{m n}^{(2)}+i \eta D_{2} \\
\delta_{\eta} D_{2} & =\partial_{m}\left(\overline{\eta \sigma}^{m} \lambda_{(2)}+h . c .\right) \\
\delta_{\eta} \phi_{2} & =\sqrt{2} \eta \psi_{2}+2 \partial_{5} a_{2} \\
\delta_{\eta} \psi_{2} & =i \sqrt{2} \sigma^{m} \bar{\eta} \partial_{m} \phi_{2}+\sqrt{2} \eta F_{2}+2 \partial_{5} \alpha_{2} \\
\delta_{\eta} F_{2} & =\partial_{m}\left(i \sqrt{2} \overline{\eta \sigma}^{m} \psi_{2}\right)+2 \partial_{5} f_{2} .
\end{aligned}
$$

One can check that on fields defined in the following way,

$$
\begin{gathered}
A_{m}=v_{m}^{(2)}, \quad \Phi+i A_{5}=-\partial_{5} C_{2}+\phi_{2} \\
\lambda_{1}=\lambda_{(2)}, \quad \lambda_{2}=\partial_{5} \chi_{2}+\frac{i}{\sqrt{2}} \psi_{2} \\
X_{3}-\partial_{5} \Phi=D_{2}, \quad X_{12}=i \partial_{5} M_{2}-F_{2}
\end{gathered}
$$


the supersymmetry transformations take the form

$$
\begin{aligned}
\delta_{\eta} A_{m} & =i \eta \sigma_{m} \bar{\lambda}_{1}+\text { h.c. }+\partial_{m} u(\eta) \\
\delta_{\eta} A_{5} & =-\eta \lambda_{2}+\text { h.c. }+\partial_{5} u(\eta) \\
\delta_{\eta} \Phi & =-i \eta \lambda_{2}+h . c . \\
\delta_{\eta} \lambda_{1} & =\sigma^{m n} \eta F_{m n}+i\left(X_{3}-\partial_{5} \Phi\right) \eta \\
\delta_{\eta} \lambda_{2} & =-i \sigma^{m} \bar{\eta} F_{m 5}-\sigma^{m} \bar{\eta} \partial_{m} \Phi-i X_{12} \eta \\
\delta_{\eta} X_{12} & =2 i \bar{\eta} \partial_{5} \bar{\lambda}_{1}-2 \overline{\eta \sigma}^{m} \partial_{m} \lambda_{2} \\
\delta_{\eta} X_{3} & =-i \eta \partial_{5} \lambda_{2}-\eta \sigma^{m} \partial_{m} \bar{\lambda}_{1}+h . c .
\end{aligned}
$$

which differs from eq. (2.8) only by the $U(1)$ gauge transformation (2.13) with

$$
u(\eta)=\eta \chi_{2}+\overline{\eta \chi}_{2}+2 \operatorname{Im}\left(a_{2}\right) .
$$

(Note that $\operatorname{Re}\left(a_{2}\right), \alpha_{2}$, and $f_{2}$ affect only the transformations of $C_{2}, \chi_{2}$, and $M_{2}$.) If it is required that we stay with the original field content, then the explicit appearance of $\chi_{2}$ is a problem. To deal with it, we can choose the extra superfield gauge transformation in a way that removes $u(\eta)$. The simplest choice that does this is given by

$$
\mathbf{\Lambda}_{\mathbf{2}}(\eta)=\left(-\frac{i}{2}\left(\eta \chi_{2}+\overline{\eta \chi}_{2}\right), \quad 0, \quad 0\right) .
$$

It affects supersymmetry transformations of only $A_{m}$ and $A_{5}$, while leaving those of $C_{2}, \chi_{2}$ and $M_{2}$ unchanged. A more involved choice,

$$
\boldsymbol{\Lambda}_{\mathbf{2}}(\eta)=\left(-i \eta \chi_{2}, \quad-\frac{i}{\sqrt{2}}\left[\sigma^{m} \bar{\eta}\left(\partial_{m} C_{2}+i A_{m}\right)+\eta M\right], \quad-i \bar{\eta} \bar{\lambda}_{1}+\overline{\eta \sigma}^{m} \partial_{m} \chi_{2}\right),
$$

makes $C_{2}, \chi_{2}$ and $M_{2}$ supersymmetry invariant. Therefore, it allows setting these fields to zero, which would put us into the Wess-Zumino gauge, $C_{2}=\chi_{2}=M_{2}=0$, while turning $\boldsymbol{\Lambda}_{\mathbf{2}}(\eta)$ into the compensating gauge transformation (2.12).

To summarize, the superfield description of the bulk 5D vector multiplet uses two $4 \mathrm{D}$ $N=1$ superfields with the following components, ${ }^{11}$

$$
\begin{aligned}
& \mathbf{V}_{\mathbf{2}}=\left(C_{2}, \quad \chi_{2}, \quad M_{2} ; \quad A_{m}, \quad \lambda_{1}, \quad X_{3}-\partial_{5} \Phi\right) \\
& \boldsymbol{\Phi}_{\mathbf{2}}=\left(\Phi+i A_{5}+\partial_{5} C_{2}, \quad-i \sqrt{2}\left(\lambda_{2}-\partial_{5} \chi_{2}\right), \quad-X_{12}+i \partial_{5} M_{2}\right) .
\end{aligned}
$$

If the superfield supersymmetry transformations do not involve a $\boldsymbol{\Lambda}_{\mathbf{2}}(\eta)$ gauge transformation, the component supersymmetry transformations differ from the ones in eq. (2.8) by a $\chi_{2^{-}}$ dependent $U(1)$ gauge transformation. The latter can be eliminated by a proper choice of $\boldsymbol{\Lambda}_{\mathbf{2}}(\eta)$. Imposing the WZ gauge corresponds to just one of many possible choices.

\footnotetext{
${ }^{11}$ This form of $\mathbf{V}_{\mathbf{2}}$ and $\boldsymbol{\Phi}_{\mathbf{2}}$ can be obtained from eq. (2.10) by a gauge transformation with the following parameter: $\boldsymbol{\Lambda}_{\mathbf{2}}=\left(\frac{1}{2} C_{2}, \frac{i}{\sqrt{2}} \chi_{2}, \frac{i}{2} M_{2}\right)$.
} 


\section{Boundary conditions for supersymmetry}

Deriving the component form of the bulk-plus-boundary action (3.7), with $\mathbf{V}_{\mathbf{2}}$ and $\mathbf{\Phi}_{\mathbf{2}}$ as in eq. (B.8), we encounter the following terms,

$$
\begin{aligned}
2 \mathbf{Z}_{\mathbf{2}}\left(\mathbf{V}_{\mathbf{2}}-\mathbf{J}\right)_{\theta^{2} \bar{\theta}^{2}} & =-\Phi\left(X_{3}-\partial_{5} \Phi-D_{J}\right)+F_{m 5}\left(A^{m}-J^{m}\right)-\left[\lambda_{2}\left(\lambda_{1}-\lambda_{J}\right)+\text { h.c. }\right] \\
& +\left(C_{2}-C_{J}\right)\left[\partial_{5}\left(X_{3}-\partial_{5} \Phi\right)-\partial_{m} \partial^{m} \Phi\right] \\
+ & {\left[\frac{i}{2} X_{12}^{*}\left(M_{2}-M_{J}\right)-\left(\partial_{5} \lambda_{1}+i \sigma^{m} \partial_{m} \bar{\lambda}_{2}\right)\left(\chi_{2}-\chi_{J}\right)+\text { h.c. }\right] . }
\end{aligned}
$$

Without the WZ gauge imposed, the boundary action then depends explicitly on the gauge degrees of freedom, $C_{2}, \chi_{2}$, and $M_{2}$. The way to eliminate them without imposing a gauge is to use a part of the boundary conditions contained in $\mathbf{V}_{\mathbf{2}} \stackrel{+0}{=} \mathbf{J}$,

$$
C_{2} \stackrel{+0}{=} C_{J}, \quad \chi_{2} \stackrel{+0}{=} \chi_{J}, \quad M_{2} \stackrel{+0}{=} M_{J}
$$

This way we arrive at the action $(4.12)$. Having used some of the boundary conditions in the action, we expect that we would need to use boundary conditions in checking supersymmetry of the simplified action. As $\left(\mathbf{V}_{\mathbf{2}}-\mathbf{J}\right)$ is a gauge invariant vector superfield, we have

$$
\delta_{\eta}\left(\mathbf{V}_{\mathbf{2}}-\mathbf{J}\right)=(\eta Q+\bar{\eta} \bar{Q})\left(\mathbf{V}_{\mathbf{2}}-\mathbf{J}\right)
$$

so that its components vary according to eq. (A.4). For example,

$$
\begin{aligned}
\delta_{\eta}\left(C_{2}-C_{J}\right) & =i \eta\left(\chi_{2}-\chi_{J}\right)+h . c . \\
\delta_{\eta}\left(\chi_{2}-\chi_{J}\right) & =\sigma^{m} \bar{\eta}\left[\partial_{m}\left(C_{2}-C_{J}\right)+i\left(A_{m}-J_{m}\right)\right]+\eta\left(M_{2}-M_{J}\right) \\
\delta_{\eta}\left(M_{2}-M_{J}\right) & =2 \bar{\eta}\left[\left(\bar{\lambda}_{1}-\bar{\lambda}_{J}\right)+i \bar{\sigma}^{m} \partial_{m}\left(\chi_{2}-\chi_{J}\right)\right] .
\end{aligned}
$$

This implies that if we use the boundary conditions for $C_{2}$ and $\chi_{2}$ in the action, then supersymmetry of the action requires using the $A_{m}$ boundary condition. Using the $M_{2}$ boundary condition leads to the $\lambda_{1}$ boundary condition. And so on. This is indeed the pattern we observed in explicit calculations. A final remark is that on-shell $X_{12}=0$, which lets us avoid using the $M_{2}$ boundary condition. This is the reason why supersymmetry of the bulk-plusboundary action (4.12) requires the use of only the $A_{m}$ boundary condition on-shell (see eq. $(5.9)$ ).

\section{References}

[1] P. Horava and E. Witten, Nucl. Phys. B 475, 94 (1996) [hep-th/9603142];

P. Horava, Phys. Rev. D 54, 7561 (1996) [hep-th/9608019].

[2] L. Randall and R. Sundrum, Phys. Rev. Lett. 83, 3370 (1999) [hep-ph/9905221];

Phys. Rev. Lett. 83, 4690 (1999) [hep-th/9906064]. 
[3] R. Altendorfer, J. Bagger and D. Nemeschansky, Phys. Rev. D 63, 125025 (2001) [hep-th/0003117]; T. Gherghetta and A. Pomarol, Nucl. Phys. B 586, 141 (2000) [hep-ph/0003129]; A. Falkowski, Z. Lalak and S. Pokorski, Phys. Lett. B 491, 172 (2000) [hep-th/0004093].

[4] E. Bergshoeff, R. Kallosh and A. Van Proeyen, JHEP 0010, 033 (2000) [hep-th/0007044].

[5] J. Bagger and D. V. Belyaev, Phys. Rev. D 67, 025004 (2003) [hep-th/0206024].

[6] D. V. Belyaev, JHEP 0601, 047 (2006) [hep-th/0509172].

[7] A. Falkowski, JHEP 0505, 073 (2005) [hep-th/0502072].

[8] E. A. Mirabelli and M. E. Peskin, Phys. Rev. D 58, 065002 (1998) [hep-th/9712214].

[9] N. Arkani-Hamed, T. Gregoire and J. Wacker, JHEP 0203, 055 (2002) [hep-th/0101233].

[10] A. Hebecker, Nucl. Phys. B 632, 101 (2002) [hep-ph/0112230].

[11] D. V. Belyaev, JHEP 0601, 046 (2006) [hep-th/0509171].

[12] W. D. Linch III, M. A. Luty and J. Phillips, Phys. Rev. D 68, 025008 (2003) [hep-th/0209060]; F. Paccetti Correia, M. G. Schmidt and Z. Tavartkiladze, Nucl. Phys. B 709, 141 (2005) [hep-th/0408138]; H. Abe and Y. Sakamura, JHEP 0410, 013 (2004) [hep-th/0408224].

[13] J. Wess and J. Bagger, Supersymmetry and Supergravity, 2nd Edition, Princeton University Press, 1992. 